\title{
Emergent reduction of electronic state dimensionality in dense ordered Li-Be alloys
}

\author{
Ji Feng $^{1} \dagger$, Richard G. Hennig ${ }^{2}$, N. W. Ashcroft ${ }^{3} \&$ Roald Hoffmann $^{1}$
}

High pressure is known to influence electronic structure and crystal packing, and can in some cases even induce compound formation between elements that do not bond under ambient conditions $^{1-3}$. Here we present a computational study showing that high pressure fundamentally alters the reactivity of the light elements lithium ( $\mathrm{Li})$ and beryllium $(\mathrm{Be})$, which are the first of the metals in the condensed state and immiscible under normal conditions ${ }^{4,5}$. We identify four stoichiometric $\mathrm{Li}_{x} \mathrm{Be}_{1-x}$ compounds that are stable over a range of pressures, and find that the electronic density of states of one of them displays a remarkable steplike feature near the bottom of the valence band and then remains almost constant with increasing energy. These characteristics are typical of a quasi-two-dimensional electronic structure, the emergence of which in a three-dimensional environment is rather unexpected. We attribute this observation to large size differences between the ionic cores of $\mathrm{Li}$ and $\mathrm{Be}$ : as the density increases, the $\mathrm{Li}$ cores start to overlap and thereby expel valence electrons into quasi-two-dimensional layers characterized by delocalized free-particle-like states in the vicinity of Be ions.

Our extensive structural search exploring possible Be-Li compound formation under pressure uses two conceptually different approaches. In the first, we propose possible structures on the basis of established chemical/physical heuristics of alloy stability at 1 atmosphere (atm) and/or high pressure. Phenomenological 1-atm binary intermetallic structure maps ${ }^{6}$ proved useful in this endeavour. In the second approach, initial structures are formed from randomly generated unit cells and atom positions, and subsequently optimized using density-functional theory $(\mathrm{DFT})^{7}$, with the random search targeting unit cells with 15 or fewer atoms. This combined staticlattice structure search explores the stoichiometries most common in binary intermetallic compounds, including $\mathrm{LiBe}, \mathrm{LiBe}_{2}, \mathrm{Li}_{2} \mathrm{Be}, \mathrm{LiBe}_{3}$, $\mathrm{Li}_{3} \mathrm{~B}, \mathrm{Li}_{2} \mathrm{Be}_{3}, \mathrm{Li}_{3} \mathrm{Be}_{2}, \mathrm{LiBe}_{4}$ and $\mathrm{Li}_{4} \mathrm{Be}$.

The enthalpy of formation of $\mathrm{Li}_{x} \mathrm{Be}_{1-x}$ is defined as $h_{\mathrm{f}}\left(\mathrm{Li}_{x} \mathrm{Be}_{1-x}\right) \equiv$ $h\left(\mathrm{Li}_{x} \mathrm{Be}_{1-x}\right)-x h(\mathrm{Li})-(1-x) h(\mathrm{Be})$, where all enthalpies $h$ are given per atom, at the same pressure, and for temperature $T \rightarrow 0$. Thermodynamically, an alloy phase is stable against decomposition to elements if its $h_{\mathrm{f}}$ is negative. The enthalpies of elements are those of the most stable known structure of elemental $\mathrm{Be}$ and $\mathrm{Li}$ at a given pressure. Elemental Be adopts the hexagonal close-packed (h.c.p.) structure $^{8}$ at low temperature in the pressure range considered, 0-200 gigapascals $(\mathrm{GPa})$, whereas $\mathrm{Li}$ in this pressure range undergoes a series of phase transitions: b.c.c. $\rightarrow$ f.c.c. $\rightarrow$ cI16 (refs 9, 10; b.c.c., body-centred cubic; f.c.c., face-centred cubic).

It is important to recognize that in these light-element phases (elements and alloys), ion dynamics can significantly change the total energies ${ }^{11}$. On the other hand, DFT static-lattice energies in general reproduce experimental phase stabilities well even for lightelement high-pressure phases ${ }^{10}$, in part owing to the cancellation of the dynamical effects in the energy differences. In the discussions that follow, we will use the static-lattice enthalpies to deduce the phase stabilities of Li-Be alloys under pressure, a procedure that is adequate in view of the large values of $h_{\mathrm{f}}$ we obtain. At finite temperatures, entropic contributions are potentially important. We estimate that the electronic ${ }^{12}$ and vibrational (Supplementary Information) entropic contribution to the free energy differences between the various Li-Be phases is of the order of $\sim 10 \mathrm{meV}$ per atom at $300 \mathrm{~K}$, about an order of magnitude smaller than the calculated ground state enthalpies of formation for the most stable phases.

Figure 1 shows the static-lattice enthalpies of formation per atom at selected pressures for the most competitive phase at each stoichiometry examined. Note that according to the definition of $h_{\mathrm{f}}$ and the tie line (Fig. 1 inset), a phase is thermodynamically stable if no tie line passes below it (multiple stable phases with different compositions can occur at the same pressure). Which phase will form in experimental synthesis will depend on the ratio of Li:Be loaded in the highpressure cells, and on other experimental factors. Kinetic effects and metastability are not considered at this level.

Our calculations indicate that for all these Li-Be ordered alloy phases, the $h_{\mathrm{f}}$ values are significantly positive $(>99 \mathrm{meV}$ per atom, see Supplementary Information) at pressure $p=0$, consistent with the fact that no compound or alloy forms at $1 \mathrm{~atm}$. But on initial compression, the $h_{\mathrm{f}}$ values of all stoichiometries drop rapidly. At around $15 \mathrm{GPa}$, the computed $h_{\mathrm{f}}$ of the most stable $\mathrm{LiBe}_{2}$ ordered alloy is already zero, and reaches a value $37 \mathrm{meV}$ per atom lower than that of the separated elements at $20 \mathrm{GPa}$ (Fig. 1). At the latter pressure, $\mathrm{LiBe}_{4}$ and $\mathrm{LiBe}$ are also stable compared to the elements, but are unstable to decomposition into $\mathrm{LiBe}_{2}$ and the corresponding elements, as indicated by the tie lines in Fig. 1.

Beyond $20 \mathrm{GPa}$, the $h_{\mathrm{f}}$ values of all Li-Be compounds continue to decrease rapidly with increasing pressure. At $80 \mathrm{GPa}$, the $h_{\mathrm{f}}$ of the most stable phase of each stoichiometry is substantially negative, with values for $\mathrm{LiBe}_{4}, \mathrm{LiBe}_{2}, \mathrm{Li}_{2} \mathrm{Be}_{3}$ and $\mathrm{LiBe}$ all below $-100 \mathrm{meV}$ per atom. The $h_{\mathrm{f}}$ of $\mathrm{LiBe}_{2}$ is especially low, at $-159 \mathrm{meV}$ per atom. As indicated by the tie lines, at $80 \mathrm{GPa}, \mathrm{LiBe}_{4}, \mathrm{LiBe}_{2}$ and $\mathrm{LiBe}$ are stable, and are expected to be accessible to experimental synthesis in view of their substantially negative heats of formation. Above $80 \mathrm{GPa}$, the $h_{\mathrm{f}}$ values of the stable $\mathrm{Li}-\mathrm{Be}$ alloys change rather slowly with pressure. At $160 \mathrm{GPa}$, we can see that only $\mathrm{LiBe}_{4}$ and $\mathrm{LiBe}_{2}$ are stable, and that $\mathrm{LiBe}$ is unstable to decomposition to $\mathrm{LiBe}_{2}$ and $\mathrm{Li}$.

The pressure ranges in which the four Li-Be phases are computed to be stable are depicted in Fig. 2a. Phonon calculations for $\mathrm{LiBe}_{4}$, $\mathrm{LiBe}_{2}$ and $\mathrm{LiBe}$ at $80 \mathrm{GPa}$ reveal no imaginary frequencies, indicating these phases possess stable (local) minima. The most stable $\mathrm{Li}_{3} \mathrm{Be}$ phase has a soft mode, which leads to a unit cell doubling with a stabilization of less than $2 \mathrm{meV}$ per atom. 
The structures of the stable $\mathrm{Li}-\mathrm{Be}$ ordered alloy phases at around $80 \mathrm{GPa}$ are displayed in Fig. 2b-e. The $\mathrm{LiBe}_{4}$ phase (Fig. 2b) has bilayers of puckered graphene-like sheets that sandwich Li atoms between them, with $\mathrm{Li}$ atoms capping the puckered hexagons. In $\mathrm{LiBe}_{2}$ (Fig. 2c), alternating Be and Li graphene-like sheets sandwich Be atoms. LiBe again has layers of puckered square nets of Li alternating with layers of puckered triangular Be nets (Fig. 2d). We emphasize that we use 'layer' as a structural descriptor; for these three phases, the interatomic distances within each layer and between layers are comparable ( $\sim 2 \AA$ at this pressure). The layering, viewed in a three-dimensional context, is quite unlike, for example, graphite, which has a larger separation between layers $(\sim 3.4 \AA)$ than within each layer $(\mathrm{C}-\mathrm{C}$ bonds $\sim 1.42 \AA$ ). Finally, we show one view of the stable monoclinic $\mathrm{BeLi}_{3}$ phase (Fig. 2e); zigzag chains of Be atoms run through channels formed by the Li host.

To understand the electronic factors governing the stability of these high-pressure Li-Be alloys and the role of crystal symmetry and geometrical structure factors, we gain some guidance by simulating the expected forms of integrated X-ray diffraction patterns ${ }^{11,13,14}$ of the four stable phases at around $80 \mathrm{GPa}$. For a range of reciprocal lattice vectors, relative peak heights can be obtained from a knowledge of the atomic scattering factors of $\mathrm{Li}$, or $\mathrm{Be}$, and the location of the associated basis vectors within the cell of the selected structure (Fig. 3a). Each of the four stable phases shows a manifold of strong diffraction peaks clustered around a narrow range of wavevectors and noticeably stronger (by at least an order of magnitude) than all other peaks. It is within these prominent clusters that $2 k_{\mathrm{F}}$ invariably lies (here $k_{\mathrm{F}}$ is the free-electron Fermi wavevector). As the diffraction intensity approximately links to the strength of a Fourier component of a self-consistent screened one-electron crystal

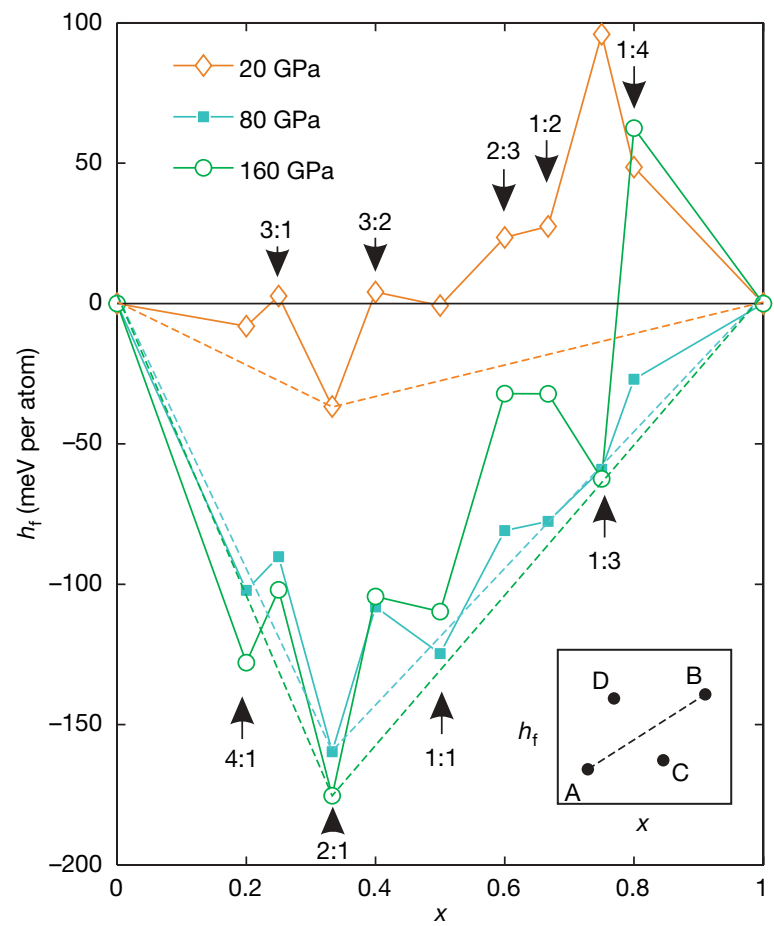

Figure 1 | Computed relative enthalpy diagram of $\mathrm{Li}_{x} \mathrm{Be}_{1-x}$ ordered alloys. The abscissa $x$ is the fraction of $\mathrm{Li}$ in the alloy, and the ordinate $h_{\mathrm{f}}$ is the enthalpy of formation per atom. Arrows indicate the Li:Be ratios. At each pressure shown, tie lines are drawn between the $\mathrm{LiBe}_{2}$ phase, which has the most negative $h_{\mathrm{f}}$, and the separated elements. Depicted schematically in the inset at lower-right is the tie line construction on a $x-h_{\mathrm{f}}$ phase diagram at a given pressure. A tie line (the dashed line) is drawn to connect phases $\mathrm{A}$ and $B$. If a third phase, such as $\mathrm{C}$, lies below the tie line, it follows then from basic thermodynamic definitions that $A$ should react with $B$ to form $C$, given there is no other competing phase. Conversely, phase $\mathrm{D}$, which lies above the A-B tie line, is expected thermodynamically to decompose into A and B. potential in the electronic case, this observation hints at a HumeRothery stabilization mechanism ${ }^{11,14-17}$; that is, these structures enjoy relatively large electronic stabilization through the interaction between an initially free-electron Fermi surface and the Brillouinzone planes associated with the strongest Fourier components. This can be seen, for example, from the massive pseudogap into which the Fermi level falls in the electronic density of states (DOS, or $g$ ) of LiBe at $82 \mathrm{GPa}$ (Fig. 3b).

However, there is something particularly unusual about the valence electronic DOS of $P 2_{1} / m$ LiBe at about $82 \mathrm{GPa}$ (see Supplementary Information for the band structure), and of a metastable $\mathrm{LiBe}_{2}$ (Pmma) phase at the same pressure (Fig. 3b). The structure of the $P m m a \mathrm{LiBe}_{2}$ (Fig. $3 \mathrm{~b}$ inset) is closely related to the $P 2_{1} / m$ LiBe structure, the only structural difference being the existence of two puckered triangular sheets in every Be layer in the former compound, instead of only one in the latter. This $\mathrm{LiBe}_{2}$ structure does not lead to a minimum enthalpy phase (for that see Fig. 2c). It is included here as a 'tuning probe', to gauge the effect of Be layer thickness.

In both compounds the bottom of the DOS of the valence band displays a striking step-function-like singularity, with a width less than $0.1 \mathrm{eV}$. After the sharp step, the DOS remains essentially constant as energy increases but is then followed by a steep rise at about $4 \mathrm{eV}$ above the bottom of the valence band, after which more distinct features emerge. The sharp step-like features in the DOS are suggestive of a van Hove singularity of a two-dimensional electronic structure, which is quite common in layered crystals (see, for example, ref. 18) but highly unusual for a compound of two metallic elements. The flat DOS places a more stringent constraint on the dispersion relation of electronic Bloch states, namely, a separable form $\varepsilon\left(k_{x}\right)+\varepsilon\left(k_{y}\right)$, where $\mathbf{k}$ is a wavevector of Bloch states in a crystal, $\varepsilon$ is the energy of an electronic state (and is quadratic in wave-vector), and the $x-y$ plane is the plane in which the two-dimensional character prevails. These features are especially remarkable considering the manifestly three-dimensional geometric structure of these ordered alloys.
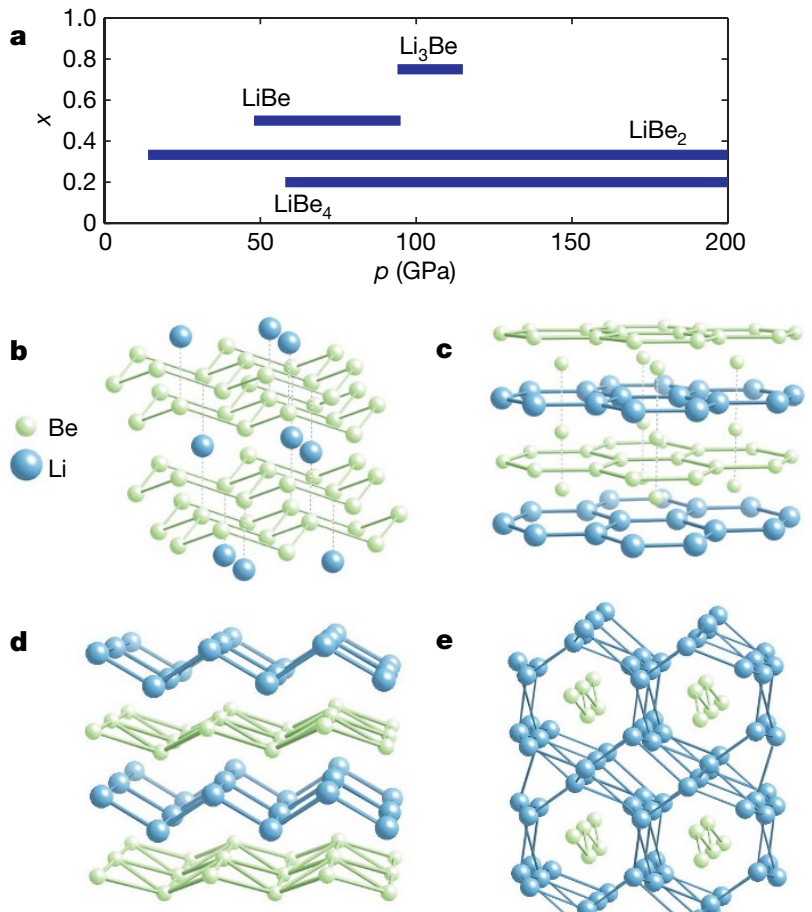

Figure 2 | Structure of stable high-pressure Li-Be compounds. Shown are $\mathrm{Be}$-Li ordered alloys predicted to be stable. a, Stability range of the four stable $\mathrm{Li}_{x} \mathrm{Be}_{1-x}$ alloys. b, $\mathrm{LiBe}_{4}$ at $80 \mathrm{GPa}$ (space group $R-3 m$ ). c, $\mathrm{LiBe}_{2}$ at $83 \mathrm{GPa}(P 6 / \mathrm{mmm})$. d, LiBe at $82 \mathrm{GPa}\left(P 2_{1} / \mathrm{m}\right)$. e, $\mathrm{Li}_{3} \mathrm{Be}$ at $80 \mathrm{GPa}(C 2 / \mathrm{m})$. Detailed structural information on these important phases is given in Supplementary Information. 
To understand how such effective electronic two-dimensionality could arise in a material that by structural criteria is patently threedimensional, we propose the following model hamiltonian in the limit of non-interacting electrons;

$$
\begin{aligned}
H= & \sum_{\mathrm{k}} \frac{\hbar^{2} k^{2}}{2 m^{*}} c_{\mathrm{k}}^{\dagger} c_{\mathrm{k}}+\sum_{2 i} \varepsilon_{o} n_{2 i}+\sum_{2 i+1}\left(\varepsilon_{o}+W\right) n_{2 i+1}+ \\
& \sum_{i}-t\left(c_{i}^{\dagger} c_{i+1}+c_{i+1}^{\dagger} c_{i}\right)
\end{aligned}
$$

where the first term describes the in-plane $(x-y)$ levels of a twodimensional free-electron gas (where the ks have no components in the $z$-direction), and the second and third terms embed a potential energy difference, $W>0$, between Be and Li layers with site indices $n_{i}$ (odd-numbered sites have higher energy). In equation (1), $m^{*}$ is the electronic effective mass, and $c$ and $c^{\dagger}$ are annihilation and creation operators. The last term describes the hopping perturbation between neighbouring Be and Li layers, characterized by a hopping energy, $t>0$. The above hamiltonian can be solved exactly; in the limit $W \gg t$, it yields a DOS as shown in Fig.3b top inset, where $\tau \equiv t^{2} / W$.

In spite of the simplicity of this hamiltonian, it reproduces the essential feature of the lower valence DOS, computed using a DFT method that in principle includes full atomic potentials and electron-electron interactions. Moreover, in Fig. $3 \mathrm{~b}$ left inset we see that the Be layers of the metastable $\mathrm{LiBe}_{2}$ are twice as thick as in the LiBe phase, and the step in DOS of $\mathrm{LiBe}_{2}$ occurs at a lower energy with roughly half the height of the LiBe case. This indicates that the two-dimensional states are associated with the Be layers. Hence, the high-potential-energy sites are associated with the Li layers, and correspondingly, the Be layers can be pictured as twodimensional potential wells.
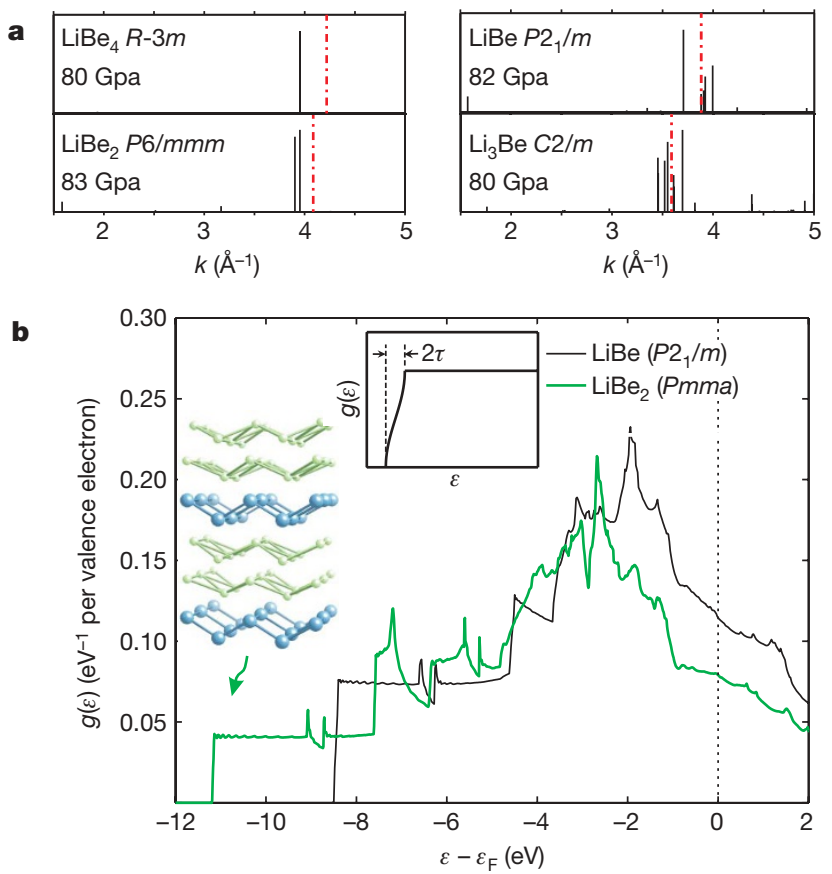

Figure 3 | Simulated X-ray diffraction patterns and electronic structures of stable $\mathrm{Li}_{1-x} \mathrm{Be}_{\mathbf{x}}$ high-pressure phases. a, Approximate X-ray diffraction patterns simulated for the four stable $\mathrm{Li}$-Be phases at around $82 \mathrm{GPa}$. Shown are the relative heights expected for the diffraction peaks, each associated with a reciprocal lattice vector belonging to the selected structure. The dashdotted lines indicate where twice the free-electron Fermi wavevector lies. b, The electronic density of states ( $g$ or DOS) of $P 2_{1} / m \mathrm{LiBe}(82 \mathrm{GPa})$ and of the structurally related metastable Pmma ( $83 \mathrm{GPa}$ ) phase. Note that the DOS is given as an intensive property, that is, in units per $\mathrm{eV}$ per valence electron. Only the valence densities of states are shown. The dotted line indicates the Fermi level. Left inset, structure of Pmma LiBe 2 . Top inset, the DOS deduced from a model hamiltonian (see text).
Figure 4 gives the computed electron density on a cross-section $(6.4 \AA \times 16.2 \AA)$ of the stable LiBe phase at $82 \mathrm{GPa}$. Outside the ionic core regions, a sharp separation is apparent between high-electrondensity zones associated with the Be layers and low-electron-density zones associated with the Li layers. There are three density extrema outside the nuclear region: $\mathrm{a}, \mathrm{b}$ and $\mathrm{c}$. Extremum a is a maximum $\left(\sim 0.059 e / a_{\mathrm{o}}{ }^{3}\right.$, where $e$ is the charge on an electron and $a_{\mathrm{o}}$ is the Bohr radius), from which the electron density drops slowly toward the nearest Be atom, but then very rapidly toward the nearest $\mathrm{Li}$ atom. Extrema b and $\mathrm{c}\left(<0.015 e / a_{\mathrm{o}}{ }^{3}\right)$ are in the middle of the shortest $\mathrm{Li}-\mathrm{Li}$ separations in the crystal (the Li atoms near $\mathrm{b}$ are above and below the plane shown). These findings are entirely consistent with the twodimensional electron gas model described by our model hamiltonian, and confirm that the two-dimensional electron gas states are clearly associated with Be layers.

In principle, the approximate segregation of valence electrons into high- and low-density regions could arise from the potential energy difference between $\mathrm{Li}$ and Be layers, as characterized by $W$ in the model hamiltonian (equation (1)). The hopping energy $t$ for $\mathrm{Li}$ and $\mathrm{Be}$ has a typical value of $\sim 1-2 \mathrm{eV}$ (Methods). The very small value of $\tau(\sim 0.05 \mathrm{eV})$ in the computed DOS indicates that $W$ is at least $\sim 20 \mathrm{eV}$, and hence much large than $t$. More importantly, a large value of $W$ indicates that there is a very large effective electronegativity difference between $\mathrm{Li}$ and Be. But the first ionization potential of Be is only about $4 \mathrm{eV}$ greater than that of $\mathrm{Li}$, which is insufficient to produce the narrow steps at the bottom of the valence electron DOS obtained with density functional theory, and it does not explain the second step at about $4 \mathrm{eV}$ above the bottom of valence bands in both $P m m a \mathrm{LiBe}_{2}$ and $P 2_{1} / m$ LiBe (Fig. 3b).

We suggest that the enhanced electronegativity differential arises because the potential energy difference between Be and Li increases under compression as a result of differential core overlap between $\mathrm{Be}$ and Li layers. Be and Li have nuclear charges of $+4 e$ and $+3 e$, respectively, so the $1 s$ core of Be has a significantly smaller spatial extent than the Li $1 s$ orbital. At $80 \mathrm{GPa}$ in BeLi, both Be-Be and Li-Li bonds are between 1.9 and 2.0 $\mathrm{A}$. In fact, the electron density of a bare $\mathrm{Li}^{+}$at $0.95 \AA$ away from the nucleus is 15 times that of a bare $\mathrm{Be}^{2+}$ (for hydrogenic wavefunctions). The difference in the spatial extent of core density is significant and even more pronounced for the common cations of these elements, whose ionic radii are $0.27 \AA\left(\mathrm{Be}^{2+}\right)$ and $0.76 \AA\left(\mathrm{Li}^{+}\right)$. In LiBe, the $\mathrm{Li}$ atomic cores have thus started to overlap significantly while the Be cores have not. Indeed, in the DFT derived band structure, the Li $1 s$ bands show a dispersion of over $1 \mathrm{eV}$, while those of Be remain within $0.1 \mathrm{eV}$. Substantial core overlap between $\mathrm{Li}$ atoms $\mathrm{s}^{9,10,17}$ results in an even higher potential energy for valence electrons, which evade the Li layers and move into the neighbourhood of Be atoms where they form almost ideal twodimensional free-electron-like states. With the enhanced electron transfer between Be and Li, our high-pressure LiBe alloys resemble

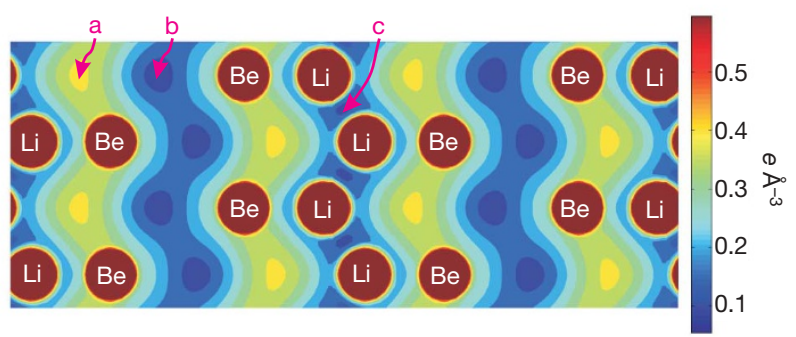

Figure 4 | Electron density of the stable LiBe phase at $82 \mathrm{GPa}$. The structure is slightly symmetrized so that we can show a plane with many atoms. The structural change in symmetrization is very small; the energy and electronic structure are essentially unchanged. Note the horizontal direction here is the direction in which $\mathrm{Li}$ and Be layers alternate, which corresponds to the vertical direction in Fig. 2d. a, b and c are density extrema (see text). 
Zintl-type ${ }^{19}$ compounds stabilized by electron transfer and the subsequent new bond formation.

We conclude by noting that because of its increased density of states at the Fermi energy compared particularly to elemental Be, and in view of the high dynamical energy scales of $\mathrm{Li}$ and Be (both superconductors in their own right), the alloy LiBe in particular may well merit assessment for superconductivity — as indeed might the other anisotropic alloys, which may even favour multi-gap pairing.

\section{METHODS SUMMARY}

We calculate the static-lattice internal energies of both Be-Li ordered alloys and the elements at given densities using the electronic DFT method ${ }^{20,21}$ within the generalized-gradient approximation, using the Perdew-Berke-Ernzerhof exchange-correlation functional ${ }^{22}$ as implemented in the Vienna ab initio Simulation Package ${ }^{23}$. At high density, the system is entering a regime of core overlap, and accordingly we perform all-electron calculations using a plane-wave basis set within the projector-augmented wave method $^{24}$. For each structure, we optimize the lattice parameters and atom positions at fixed volumes, using a conjugated-gradient algorithm based on Hellmann-Feynman forces. At each volume, the structural optimization is done twice, the second starting with the optimized geometry from the first run. The optimized geometry of the various elemental phases is in good agreement with the experimental values. (For example, the equilibrium lattice constants of the b.c.c. Li and the h.c.p. Be agree with experimental values to $<3 \%$. Also, the calculated atomic volume of the $c I 16$ $\mathrm{Li}$ at $44 \mathrm{GPa}\left(8.75 \AA^{3}\right)$ and fractional coordinates of the atom $(x, x, x)$ with $x=0.051$ compare very well with the powder diffraction data, $8.8 \AA^{3}$ and $x \approx 0.052$; ref. 10.) Then a single-point calculation is performed to evaluate the internal energy based on the pre-optimized geometry. The energy cut-off and k-point sampling are chosen such that energies are convergent within $\sim 3 \times 10^{-3} \mathrm{eV}$ per atom over the pressure range. From calculated internal energy as a function of unit cell volume, $E(V)$, the ground state enthalpy $h$ and hence $h_{\mathrm{f}}$ can be deduced from $h \equiv(E+p V) / N$, where $N$ is the number of atoms, and the pressure $p \equiv-\mathrm{d} E / \mathrm{d} V$. Phonon calculations are carried out using VASP combined with $\mathrm{PHON}^{25}$ on supercells of around 100 atoms. We use the extended Hückel method for estimating the hopping energy between Li and Be layers ${ }^{26}$. Symmetry identification of the random-search structures was carried out using ISOTROPY (http://stokes.byu.edu/isotropy.html).

\section{Received 3 July; accepted 25 October 2007.}

1. Parker, L. J., Atou, T. \& Badding, J. V. Transition element-like chemistry for potassium under pressure. Science 273, 95-97 (1996).

2. Atou, T., Hasegawa, M., Parker, L. J. \& Badding, J. V. Unusual chemical behaviour for potassium under pressure: Potassium-silver compounds. J. Am. Chem. Soc. 118, 12104-12108 (1996)

3. Tsvyashchenko, A. V. et al. New ferromagnetic compound $\mathrm{CaCO}_{2}(\mathrm{C} 15)$ synthesized at high pressure. JETP Lett. 68, 908-914 (1998).

4. Hansen, M. Constitution of Binary Alloys (McGraw-Hill Book Company, Inc., New York, 1958).

5. Massalski, T. B., Murray, J. L., Bennett, L. H. \& Baker, H. Binary Alloy Phase Diagrams Vol. 1 460-461 (American Society for Metals, Metals Park, 1986).
6. Pettifor, D. G. The structures of binary compounds: I. Phenomenological structure maps. J. Phys. C 19, 285-313 (1986).

7. Pickard, C. J. \& Needs, R. J. High-pressure phases of silane. Phys. Rev. Lett. 97, 045504 (2006)

8. Robert, G. \& Sollier, A. Equations of state and elastic properties of beryllium from first principles calculations. J. Phys. IV 134, 257-262 (2006).

9. Neaton, J. B. \& Ashcroft, N. W. Pairing in dense lithium. Nature 400, 141-144 (1999).

10. Hanfland, M., Syassen, K., Christensen, N. E. \& Novikov, D. L. New high-pressure phases of lithium. Nature 408, 174-178 (2000).

11. Ashcroft, N. W. Quantum-solid behaviour and the electronic structure of the light alkali metals. Phys. Rev. B 39, 10552-10559 (1989).

12. Watson, R. E. \& Weinert, M. Contribution of electronic excitations to the entropy of crystals: The relative stabilities of the hcp, fcc, and bcc structures among the transition metals. Phys. Rev. B 30, 1641-1645 (1984).

13. Kraus, W. \& Nolze, G. POWDER CELL - a program for the representation and manipulation of crystal structures and calculation of the resulting X-ray powder patterns. J. Appl. Crystallogr. 29, 301-303 (1996).

14. Ackland, G. J. \& Macleod, I. R. Origin of the complex crystal structures of elements at intermediate pressure. New J. Phys. 6, 138 (2004)

15. Mott, N. F. \& Jones, H. The Theory of the Properties of Metals and Alloys 152-174 (Dover, New York, 1958).

16. Degtyareva, V. F. Simple metals at high pressures: The Fermi sphere-Brillouin zone interaction model. Phys. Usp. 49, 369-388 (2006).

17. Feng, J., Ashcroft, N. W. \& Hoffmann, R. Theoretical indications of singular structural and electronic features of Laves-phase $\mathrm{CaLi}_{2}$ under pressure. Phys. Rev. Lett. 98, 247002 (2007).

18. Kolmogorov, A. N. \& Curtarolo, S. Prediction of different crystal structure phases in metal borides: A lithium monoboride analog to $\mathrm{MgB}_{2}$. Phys. Rev. B 73, 180501 (2006).

19. Corbett, J. D. in Chemistry, Structure, and Bonding of Zintl Phases and lons (ed. Kauzlarich, S. M.) 139-182 (Wiley-VCH, New York, 1996).

20. Hohenberg, P. \& Kohn, W. Inhomogeneous electron gas. Phys. Rev. 136, B864-B871 (1964).

21. Kohn, W. \& Sham, L. J. Self-consistent equations including exchange and correlation effects. Phys. Rev. 140, A1133-A1138 (1965).

22. Perdew, J. P., Burke, K. \& Ernzerhof, M. Generalized gradient approximation made simple. Phys. Rev. Lett. 77, 3865-3868 (1996).

23. Kresse, G. \& Hafner, J. Ab initio molecular dynamics for liquid metals. Phys. Rev. $B$ 47, 558-561 (1993).

24. Kresse, G. \& Joubert, J. From ultrasoft pseudopotentials to the projector augmented wave method. Phys. Rev. B 59, 1758-1775 (1999).

25. Vocadlo, L., Alfè, D., Brodholt, J., Gillan, M. J. \& Price, G. D. The structure of iron under the conditions of the Earth's inner core. Geophys. Res. Lett. 26, 1231-1235 (1999).

26. Hoffmann, R. An extended Hückel theory. I. Hydrocarbons. J. Chem. Phys. 39, 1397-1412 (1963).

Supplementary Information is linked to the online version of the paper at www.nature.com/nature.

Acknowledgements We thank the National Science Foundation and the Petroleum Research Fund of the American Chemical Society for support of this work, and A. Bergara, P. Kroll, W. Grochala and K. Donald for comments.

Author Information Reprints and permissions information is available at www.nature.com/reprints. Correspondence and requests for materials should be addressed to N.W.A. (nwa@ccmr.cornell.edu) and R.H. (rh34@cornell.edu). 